Table 1. List of Procedures Based on Risk for Aerosol Generation

\begin{tabular}{ll}
\hline Risk & \multicolumn{1}{c}{ Procedure } \\
\hline Low-risk AGP & Nasopharyngeal/oropharyngeal sample collection \\
& Metered-dose inhaler \\
& Insertion of nasogastric/orogastric tube \\
& Closed or nasal/oropharyngeal suctioning \\
\hline High-risk ${ }^{\text {a AGP }}$ & Open airway deep or tracheal suctioning (below \\
& vocal cords) \\
& Mechanical ventilation \\
& Tracheal intubation or extubation \\
& Laryngeal mask airway \\
& Noninvasive ventilation (BiPAP, CPAP, HFNC) \\
& Tracheostomy placement/patient with tracheostomy \\
& Cardiopulmonary resuscitation \\
& Bronchoscopy \\
& Sputum induction/cough assist \\
& Nebulizer therapy \\
& Cool mist humidification \\
\hline
\end{tabular}

Note. BiPAP, bilevel positive airway pressure; CPAP, continuous positive airway pressure; HFNC, high-flow nasal cannula.

${ }^{a}$ N95/CAPR recommended only for high-risk aerosol-generating procedures.

successfully limited N95 misuse as community COVID-19 burden increased, despite rising inpatient volumes of confirmed COVID-19 and enacting universal COVID-19 admission testing, which required $\sim 8$-24 hours of COVID-19 isolation for all inpatients pending test results.

Following the intervention, IP\&C met with hospital units and departments to discuss successes and areas for improvement in our ongoing hospital COVID-19 response. A consistent theme was that the PPE Spotter team was very well received and fulfilled the intended educational and PPE preservation goals. Stakeholders identified IP\&C and the PPE Spotter team as an excellent multidisciplinary resource, particularly during periods of evolving PPE guidance and supply chain insecurity. An additional benefit of this team was further expanding the pool of hospital staff to advocate for HCW safety and IP\&C principles. We are working to determine how we can continue a similar model as COVID-19 activity decreases to maintain a readily available resource to bedside healthcare providers. In summary, our stewardship initiatives for PPE were successful in minimizing PPE misuse, and they provided a conduit for real-time education to frontline providers.

Acknowledgments. None.

Financial support. No financial support was provided relevant to this article.

Conflicts of interest. All authors report no conflicts of interest relevant to this article.

\section{References}

1. Lynch JB, Davitkov P, Anderson DJ, et al. Infectious Diseases Society of America guidelines on infection prevention in patients with suspected or known COVID-19. Infectious Diseases Society of America website. https:// www.idsociety.org/practice-guideline/covid-19-guideline-infection-prevention/. Published April 27, 2020. Accessed June 24, 2020.

2. Tran K, Cimon K, Severn M, Pessoa-Silva CL, Conly J. Aerosol-generating procedures and risk of transmission of acute respiratory infections to healthcare workers: a systematic review. PLoS One 2012;7(4):e35797.

\title{
Coronavirus disease 2019 (COVID-19) among healthcare workers: A call for a low-threshold in-hospital screening
}

\author{
Hytham K. S. Hamid MRCSEd (10 \\ Department of Surgery, Soba University Hospital, Khartoum, Sudan
}

To the Editor-A recent large study have shown that only $3 \%$ of healthcare workers (HCWs) infected with the novel severe acute respiratory coronavirus virus 2 (SARS-CoV-2) have been exposed to an inpatient with coronavirus disease 2019 (COVID-19) prior to the onset of symptoms, suggesting that most HCWs acquire infection in the community, perhaps due to contact with presymptomatic or asymptomatic carriers, rather than in hospital settings. ${ }^{1}$ The high prevalence of infection (11\%-20\%) among HCWs supports this assertion, ${ }^{2,3}$ despite the reportedly low risk of nosocomial infection associated with SARS-CoV-2., ${ }^{1,4}$

Wee et $\mathrm{al}^{6}$ recently reported their experience with syndromic surveillance of HCWs for COVID-19. The surveillance was based on symptoms of acute respiratory illness (ARI) and fever. Pointing

Author for correspondence: Hytham K. S. Hamid, E-mail: kujali2@gmail.com

Cite this article: Hamid HKS. (2021). Coronavirus disease 2019 (COVID-19) among healthcare workers: A call for a low-threshold in-hospital screening. Infection Control \& Hospital Epidemiology, 42: 637-638, https://doi.org/10.1017/ice.2020.324 to community as well as in-hospital secondary transmission, these researchers detected 14 cases of COVID-19 among HCWs with 4 clusters; most were linked via transmission outside of hospital. ${ }^{6}$ However, although syndromic surveillance, based on fever and ARI symptoms, was shown to be effective during the outbreak of severe acute respiratory syndrome (SARS), ${ }^{7}$ it may not be as effective in containment of COVID-19 infection because of different patterns of clinical presentations. Indeed, in the study by Wee et al, ${ }^{6}$ heat maps did not pick up the cluster with suspected intrahospital spread. Moreover, in a study by Tostmann et $\mathrm{al}^{2}$ employing a low-threshold screening for SARS-CoV-2, most HCWs with SARS-CoV-2 infection had mild clinical presentations, frequently not including fever or respiratory symptoms. Using the same clinical data, these researchers developed a model, excluding fever and cough, to predict COVID-19 among HCWs with a fair discriminative ability. ${ }^{2}$

Early identification and control of COVID-19 among HCWs is of paramount importance particularly in the postoutbreak period to 
prevent in-hospital secondary transmission to other HCWs and inpatients. The concern of transmitting infection into the healthcare system has been highlighted in a recent study in which HCWs were linked to transmission of COVID-19 into long-term healthcare facilities. ${ }^{8}$ Considering that as many as $50 \%$ of all SARS-CoV-2 infections are asymptomatic, ${ }^{9}$ it would seem appropriate, when resources are available, to perform routine SARS-CoV-2 nasopharyngeal screening for all HCWs. The prediction model described by Tostmann et al, ${ }^{2}$ rather than fever and ARI symptoms, can be used to guide a targeted screening strategy in settings with limited availability of testing materials.

Acknowledgments. None.

Financial support. No financial support was provided relevant to this article.

Conflicts of interest. All authors report no conflicts of interest relevant to this article.

\section{References}

1. Kluytmans M, Buiting A, Pas S, et al. SARS-CoV-2 infection in 86 healthcare workers in two Dutch hospitals in March 2020. MedRxiv 2020. doi: 10.1101/ 2020.03.23.20041913.
2. Tostmann A, Bradley J, Bousema T, et al. Strong associations and moderate predictive value of early symptoms for SARS-CoV-2 test positivity among healthcare workers, the Netherlands, March 2020. Euro Surveill 2020;25:2000508.

3. Lo D. COVID-19: protecting healthcare workers. Lancet 2020;395:922.

4. Cheng V, Wong S-C, Chen J, et al. Escalating infection control response to the rapidly evolving epidemiology of the coronavirus disease 2019 (COVID19) due to SARS-CoV-2 in Hong Kong. Infect Control Hosp Epidemiol 2020;41:493-498.

5. Ng K, Poon BH, Kiat Puar TH, et al. COVID-19 and the risk to healthcare workers: a case report. Ann Intern Med 2020;172:766-767.

6. Wee LE, Sim XYJ, Conceicao EP, et al. Containment of COVID-19 cases among healthcare workers: the role of surveillance, early detection, and outbreak management. Infect Control Hosp Epidemiol 2020 May 11 [Epub ahead of print]. doi: 10.1017/ice.2020.219.

7. Tan CC. SARS in Singapore-key lessons from an epidemic. Ann Acad Med Singapore 2006;35:345-349.

8. McMichael TM, Currie DW, Clark S, et al. Epidemiology of COVID-19 in a long-term care facility in King County, Washington. $N$ Engl J Med 2020;382:2005-2011.

9. Graham LA, Maldonado YA, Tompkins LS, Wald SH, Chawla A, Hawn MT. Asymptomatic SARS-CoV-2 transmission from community contacts in healthcare workers. Ann Surg 2020 (in press).

\title{
The prevalence of severe acute respiratory coronavirus virus 2 (SARS-CoV-2) IgG antibodies in intensive care unit (ICU) healthcare personnel (HCP) and its implications - a single-center, prospective, pilot study
}

\author{
Mohsin Sheraz Mughal MBBS, Ikwinder Preet Kaur MBBS, Chandler D. Patton MD, Nagy H. Mikhail MD, \\ Chairut Vareechon PhD and Kenneth M. Granet MD, FACP \\ Monmouth Medical Center, an affiliate of the RWJ/Barnabas Health System, Long Branch, New Jersey
}

To the Editor-Healthcare personnel (HCP), including practitioners, nursing staff, respiratory therapists, and the pronepositioning team caring for coronavirus disease 2019 (COVID-19) patients in the intensive care unit (ICU) are considered to have a high risk of exposure to severe acute respiratory syndrome coronavirus 2 (SARS-CoV-2). Most patients admitted to the ICU are severely sick and need to be intubated. High-risk procedures for droplet dispersion, including tracheal intubation and tracheostomy tube placement, can be performed in the ICU. ${ }^{1}$ In a community seroprevalence study in Los Angeles County, the prevalence of antibodies to SARS-CoV-2 was $4.65 \%{ }^{2}$ To our knowledge, no other study has addressed the prevalence of subclinical seroconversion of SARSCoV-2 among HCP in the ICU setting. In this study, we investigated the seroconversion of asymptomatic SARS-CoV-2 infection in ICU HCP exposed to critically ill COVID-19 patients.

Author for correspondence: Kenneth M. Granet, E-mail: Kenneth.Granet@rwjbh.org. Cite this article: Mughal MS, et al. (2021). The prevalence of severe acute respiratory coronavirus virus 2 (SARS-CoV-2) IgG antibodies in intensive care unit (ICU) healthcare personnel (HCP) and its implications-a single-center, prospective, pilot study. Infection Control \& Hospital Epidemiology, 42: 638-639, https://doi.org/10.1017/ice.2020.298

\section{Methods}

This single-center, prospective, pilot study was performed in an ICU at a teaching hospital, Monmouth Medical Center in Long Branch, New Jersey. It was approved by our institutional review board. All HCP caring for COVID-19 patients in the ICU setting from March 1, 2020, to April 30, 2020, were eligible for inclusion in the study. A cross-sectional survey questionnaire was utilized to collect demographic characteristics and to exclude HCP who (1) tested positive for SARS-CoV-2 by reverse transcriptasepolymerase chain reaction assay (RT-PCR), (2) had symptoms consistent with COVID-19, or (3) had COVID-19 exposure in a household setting. In total, 134 ICU HCP responded to the survey, and $121 \mathrm{HCP}$ were eligible for SARS-CoV-2-specific IgG antibody testing. Means and interquartile ranges (IQRs) were used for continuous variables. All participants provided written consent. Antibody testing was performed on the sera using a rapid immunochromatography test (STANDARD Q COVID-19 IgM/IgG Duo, SD Biosensor, Suwon-si, Korea) by lateral flow in a Clinical Laboratory Improvement Amendments certified (CLIA), high-complexity laboratory. The manufacturer's stated 